\title{
Familial clustering of suicide risk: a total population study of 11.4 million individuals
}

\author{
D. Tidemalm ${ }^{1 *}$, B. Runeson ${ }^{1}$, M. Waern ${ }^{2}$, T. Frisell ${ }^{3,4}$, E. Carlström ${ }^{3}$, P. Lichtenstein ${ }^{3}$ \\ and N. Långström ${ }^{3,4}$ \\ ${ }^{1}$ Department of Clinical Neuroscience, Karolinska Institutet, Division of Psychiatry, Stockholm, Sweden \\ ${ }^{2}$ Institute of Neuroscience and Physiology, Section of Psychiatry and Neurochemistry, Sahlgrenska Academy at Göteborg University, \\ Göteborg, Sweden \\ ${ }^{3}$ Department of Medical Epidemiology and Biostatistics, Karolinska Institutet, Stockholm, Sweden \\ ${ }^{4}$ Centre for Violence Prevention, Karolinska Institutet, Stockholm, Sweden
}

Background. Research suggests that suicidal behaviour is aggregated in families. However, due to methodological limitations, including small sample sizes, the strength and pattern of this aggregation remains uncertain.

Method. We examined the familial clustering of completed suicide in a Swedish total population sample. We linked the Cause of Death and Multi-Generation Registers and compared suicide rates among relatives of all 83951 suicide decedents from 1952-2003 with those among relatives of population controls.

Results. Patterns of familial aggregation of suicide among relatives to suicide decedents suggested genetic influences on suicide risk; the risk among full siblings (odds ratio $3.1,95 \%$ confidence interval $2.8-3.5,50 \%$ genetic similarity) was higher than that for maternal half-siblings (1.7, 1.1-2.7, $25 \%$ genetic similarity), despite similar environmental exposure. Further, monozygotic twins (100\% genetic similarity) had a higher risk than dizygotic twins ( $50 \%$ genetic similarity) and cousins $(12.5 \%$ genetic similarity) had higher suicide risk than controls. Shared (familial) environmental influences were also indicated; siblings to suicide decedents had a higher risk than offspring (both $50 \%$ genetically identical but siblings having a more shared environment, 3.1, 2.8-3.5 v. 2.0, 1.9-2.2), and maternal half-siblings had a higher risk than paternal half-siblings (both $50 \%$ genetically identical but the former with a more shared environment). Although comparisons of twins and half-siblings had overlapping confidence intervals, they were supported by sensitivity analyses, also including suicide attempts.

Conclusions. Familial clustering of suicide is primarily influenced by genetic and also shared environmental factors. The family history of suicide should be considered when assessing suicide risk in clinical settings or designing and administering preventive interventions.

Received 3 December 2010; Revised 28 April 2011; Accepted 30 April 2011; First published online 1 June 2011

Key words: Family studies, intergenerational transmission, nationwide registers, suicidal behaviour, suicide.

\section{Introduction}

Suicide and attempted suicide are leading causes of death and morbidity worldwide. According to prognoses by the World Health Organization, approximately 1.5 million people will die from suicide in 2020 (Bertolote \& Fleischmann, 2005). Suicidal behaviour runs in families (Brent et al. 1996; Runeson, 1998; Brent \& Mann, 2005) and the familial transmission appears, at least partly, to be independent of psychiatric disorder (Agerbo et al. 2002; Qin et al. 2002, 2003; Runeson \& Åsberg, 2003; Kim et al. 2005; McGirr et al. 2009; Brezo et al. 2010).

\footnotetext{
* Address for correspondence: Dr D. Tidemalm, Department of Clinical Neuroscience, Karolinska Institutet, Division of Psychiatry, St Göran, SE-112 81 Stockholm, Sweden.

(Email : dag.tidemalm@ki.se)
}

Theoretically, familial aggregation of suicides could be explained by shared genes, shared environments or both. In fact, results from prior family, adoption, twin, molecular genetic, geographic and immigrant studies do suggest that familial clustering of suicidal acts has genetic as well as environmental causes (Turecki, 2001; Baldessarini \& Hennen, 2004; Brent \& Mann, 2005; Voracek \& Loibl, 2007, 2008; Wasserman et al. 2007). However, due to underpowered studies and limitations of data available for study, the strength and patterns of genetic and environmental impact on familial aggregation of suicidal behaviour remain uncertain (Baldessarini \& Hennen, 2004; Baud, 2005; Bondy et al. 2006; Brent \& Melhem, 2008; Brezo et al. 2008, 2010; Currier \& Mann, 2008).

Three large twin studies yielded estimates of the genetic contribution to risk of a suicide attempt

The online version of this article is published within an Open Access environment subject to the conditions of the Creative Commons Attribution-NonCommercial-ShareAlike licence < http://creativecommons.org/licenses/by-nc-sa/2.5/>. The written permission of Cambridge University Press must be obtained for commercial re-use. 
ranging from $17 \%$ (adjusted for heritability of other risk factors) to 55\% (Statham et al. 1998; Glowinski et al. 2001; Fu et al. 2002). Further, adoption studies (Kety et al. 1968; Schulsinger et al. 1979; Wender et al. 1986) have demonstrated increased risk of completed suicide in biological relatives of adopted-away probands with suicidal behaviour. These results are usually interpreted as evidence of a genetic contribution to suicide risk (Roy et al. 1997; Brent \& Mann, 2005; Ernst et al. 2009). However, these children may have been subjected to detrimental environmental effects in utero (Mittendorfer-Rutz et al. 2004; Magnusson et al. 2005; Riordan et al. 2006) or in early childhood prior to adoption (Roy, 2002; Mann et al. 2005; Currier \& Mann, 2008; Hawton \& van Heeringen, 2009). Further, a recent large Swedish adoption study of familial risk factors and suicide suggested a specific genetic contribution to suicide risk (von Borczyskowski et al. 2010).

Pooled data demonstrate a threefold risk of suicidal behaviour (suicide attempt or completed suicide) in first- and second-degree relatives of persons who attempted or died from suicide (Baldessarini \& Hennen, 2004). However, this and other meta-analyses (Turecki, 2001; Brent \& Mann, 2005) were limited by heterogeneous study designs as well as varying and usually small sample sizes. To date, a few large register-based studies of familial clustering of suicide have been published (Agerbo et al. 2002; Qin et al. 2002, 2003; Runeson \& Åsberg, 2003; MittendorferRutz et al. 2008; von Borczyskowski et al. 2010). Two of these reported a twofold risk of suicide in first-degree relatives of suicide victims after adjustment for mental disorder (Qin et al. 2003; Runeson \& Åsberg, 2003). However, none of these prior studies reported risks across different classes of first-degree relatives or in second- and third-degree relatives. Importantly, such risk estimates would increase our understanding of genetic and environmental influences on the familial aggregation of completed suicide (Brent, 2010).

We aimed to examine familial risks of completed suicide in the total population of Sweden using data from 1952-2003. We hypothesized substantial familial aggregation of suicide and that suicide risk would primarily vary by degree of genetic relatedness (Segal, 2009; Pedersen \& Fiske, 2010).

\section{Method}

\section{Subjects and measures}

We created a large database by linking three national longitudinal population registers in Sweden using the personal identity number as primary key. The latter is a unique persistent identifier assigned to the individual at birth or immigration and used for health and welfare statistics (Ludvigsson et al. 2009). The Cause of Death Register, held by the National Board of Health and Welfare, includes all deaths among persons registered as residents of Sweden at the time of death. Established in 1952, the register covers $>99 \%$ of all deaths from 1961 and onwards, including those occurring abroad (National Board of Health and Welfare, 2010). Each record contains the date of death and codes for causes of death, including definite and uncertain suicide, in accordance with ICD-6, -7, -8, -9 and -10 . The National Inpatient Register (maintained by the National Board of Health and Welfare) covers all in-patient health care (including the few existing private hospitals). The register has complete national coverage for all psychiatric admissions from 1973 and onwards. Each record contains admission and discharge dates, diagnoses and codes for attempted suicide (method used and if definite or uncertain) according to ICD-8, -9 and -10 . Diagnoses and codes for suicide attempts are determined and recorded by the physician at discharge. The Multi-Generation Register (maintained by Statistics Sweden) contains information about child-parent relationships for all children born in Sweden since 1932 and who were still alive in 1961. Swedish-born children and children who immigrated to Sweden with at least one parent and were granted citizenship before age 18 years are linked to their parents. On 31 December 2004, the register contained 7969645 index persons together with their biological and adoptive parents; in total, 11384649 individuals. From these child-parent dyads, it is possible to construct larger pedigrees, including relatives at increasing distances (genetic and environmental) from each index person.

From the Cause of Death Register, we identified all Swedish residents who died from a definite suicide (ICD-6 [1951-1957]: E970-9; ICD-7 [1958-1968]: E971.9-979.9; ICD-8 [1969-1986], ICD-9 [1987-1996]: E950-9; ICD10 [1997-]: X60-84) or an uncertain suicide (ICD-8, ICD-9: E980-9; ICD10: Y10-34) between 1 January 1952 and 31 December $2003(n=83951)$. Uncertain suicides (not coded in ICD-6 and ICD-7) were included to avoid underestimation of suicide rates (Neeleman \& Wessely, 1997). Further, we identified all cases of a definite or uncertain suicide attempt from the National Inpatient Register (ICD-8, -9 and -10 , codes correspond to those for completed suicide) between 1 January 1973 and 31 December 2003.

\section{Statistical analyses}

We used a matched case-control design to estimate familial suicide risks for different classes of relatives and other close relationships. We studied relatives at 
Table 1. Relative risks of suicide in relatives of all probands who committed suicide in Sweden during 1952-2003 ( $n=83$ 951) compared with relatives of matched controls, in a total population cohort of 11384649 individuals

\begin{tabular}{lccc|c|c|c}
\hline Relation to proband & $\begin{array}{l}\text { Number } \\
\text { of dyads }\end{array}$ & $\begin{array}{c}\text { Concordant } \\
\text { pairs }\end{array}$ & 10451878 & 486 & & Matched \\
OR (95\% CI)
\end{tabular}

Relative risks were calculated with conditional logistic regression by comparing suicide rates in each proband-relative dyad type with population control-relative dyads matched 1:5 by relative category, gender, birth year and time at risk. The graph depicts odds ratios (OR) and $95 \%$ confidence intervals (CI) for all dyads $(\bullet)$. Each individual in the population could appear multiple times in different categories (e.g. sibling, child, cousin, etc.) depending on family pedigree.

varying distances from probands who had committed suicide. For instance, for each suicide proband, we specified all possible case-sibling pairs consisting of the proband and each of his/her full siblings (who had or had not committed suicide). For each case-sibling pair, we randomly selected five control-sibling pairs matched to case pairs by gender and birth year. The controls were selected through risk set sampling; that is, individuals were eligible as controls if they were alive and living in Sweden at the time of the case person's suicide, regardless of whether they later committed suicide. This matching procedure was used for the probands' first-, second- and third-degree relatives, as well as monozygotic twins, spouses/ unrelated partners and adopted children. First-degree relatives comprised parents, full siblings (including dizygotic twins) and children. Second-degree relatives were grandparents, uncles/aunts, half-siblings, nephews/nieces and grandchildren, while thirddegree relatives consisted of cousins. To increase statistical power, suicidal behaviour (completed suicide or suicide attempt leading to hospital care) was used as a broader suicidal behaviour phenotype for classes of relatives with few pairs concordant for completed suicide.

We calculated odds ratios (OR) using conditional logistic regression in PROC PHREG in SAS version 9.2
(SAS Institute, USA). Since multiple probands were possible in each family constellation, we adjusted for the non-independence of probands by computing corrected (less narrow) confidence intervals (CI) with a robust sandwich estimator (covsandwich option in PHREG). A similar case-control method was previously applied to calculate familial risks of schizophrenia, bipolar disorder (Lichtenstein et al. 2009) and violent offending (Frisell et al. 2011). For twin analyses, asymmetric CI for proportions were calculated with PROC FREQ in SAS version 9.2 (SAS Institute).

\section{Results}

This total population sample included almost 8.0 million individuals of known parentage, which, together with their parents, constituted 11.4 million unique individuals. A total of 83951 individuals committed suicide during the study period. Table 1 shows the relative risks of suicide in biological and nonbiological relatives of probands who died by suicide compared to risks in relatives of matched control individuals. First, risk patterns suggested genetic influences on suicide risk; the risk for full siblings (OR 3.1, 95\% CI 2.8-3.5, 50\% genetic similarity) was significantly higher than that for maternal half-siblings (OR 1.7, 95\% CI 1.1-2.7, 25\% genetic similarity), 
despite similar environmental exposure. Genetic impact was also indicated by monozygotic twins (100\% genetically identical) having a substantially higher risk for completed suicide than had dizygotic twins (50\% genetic similarity), albeit with overlapping CI. Further, cousins, with $12.5 \%$ genetic similarity and low or marginal immediate environmental exposure to the suicide proband, had a $50 \%$ higher suicide risk than controls (OR 1.5, 95\% CI 1.3-1.8).

Second, shared environmental influences on suicide risk were also suggested. Full siblings had a significantly higher suicide risk than offspring of suicide decedents (both with 50\% genetic similarity but siblings having a more shared environment, OR 3.1, 95\% CI 2.8-3.5 v. OR 2.0, 95\% CI 1.9-2.2). Also, maternal half-siblings had a higher risk of suicide than paternal half-siblings (OR 1.7, 95\% CI 1.1-2.7 v. OR 1.2, 95\% CI $0.8-1.9$, both with $50 \%$ genetic similarity but the former with a more shared environment than the latter). However, this comparison had overlapping CI.

Spouses were at increased risk of suicide (OR 2.3, 95\% CI 2.2-2.5); this relative risk was higher than, for example, that for offspring. However, increased suicide risk among spouses is not necessarily caused by the family environment shared by the couple, but may also suggest assortative mating. This may occur because of environmental factors such as social class, which affect the likelihood to select a certain spouse, or because of the studied phenotype, although not necessarily being a conscious or rational choice of the mating partner. We were unable to further distinguish between the relative importance of the mechanisms that might have caused this association. Assortative mating could potentially bias heritability estimates for suicidal behaviour, but does not lead to different interpretations of the familial risks in this study (Frisell et al. in press).

Finally, as a sensitivity test, we attempted to obtain more precise risk estimates for selected comparisons based on Table 1. Hence, assuming that suicide attempts requiring in-patient care and completed suicide might be expressions of the same liability to suicidal behaviour, we calculated corresponding relative risks for suicidal behaviour (completed suicide or a suicide attempt leading to hospital care). The resulting OR were similar to those for completed suicide; however, since substantially more pairs were concordant for suicidal behaviour, CI did not overlap as much (see Appendix). Thus, with increased power we now found comparisons of monozygotic and dizygotic (same-gender) twins (OR 12.3, 95\% CI 6.523.2 v. 2.3, 1.1-5.0) statistically support genetic influences on familial aggregation of suicidal behaviour. Similarly, shared environmental influences on suicide risk were confirmed by maternal half-siblings having significantly higher suicide risk than paternal halfsiblings (OR 1.5, 95\% CI 1.3-1.7 v. OR 1.1, 95\% CI 0.91.2). Finally, genetic and shared environmental effects were both statistically supported by adopted-away biological children and adopted (non-biological) children having higher risks than controls (OR 1.6, 95\% CI 1.2-2.0 for both comparisons).

\section{Discussion}

This is the first total population study providing robust estimates of familial suicide risk in relatives at varying genetic and environmental distance from each other; including half-siblings, grandchildren, cousins and spouses. Importantly, since this investigation was four times larger than any previous study, we had the power to identify shared environmental effects on the risk of completed suicide. In line with our two hypotheses, we found evidence for familial aggregation of completed suicide influenced primarily by genetic and also by shared environmental factors. For example, we found a twofold increased risk of suicide in children and a threefold risk in siblings of suicide probands, compared with corresponding relatives of controls. These risk estimates are somewhat lower than the average size reported among first-degree relatives (children and siblings) in a smaller Danish study of a rather comparable design (Qin et al. 2003).

A few limitations should be noted. The study was based solely on register data, although with excellent coverage and validity $>40$ years. Second, longitudinal registers are subject to 'left censoring', or missing data before the date the register started. However, since we matched for birth year and time at risk, such losses would be similar for both case and control dyads and not affect the estimates of familial aggregation (i.e. the relative risks). Third, despite the large sample size, some results were probably non-significant due to insufficient statistical power to detect aggregation of uncommon events, such as completed suicides. Nevertheless, sensitivity analyses using a broader measure of suicidal behaviour including attempted suicides leading to hospitalization generally supported the main findings. Fourth, the matching procedure did not include socio-economic status. Fifth, familial risks were not easily adjusted for presence of mental disorder in relatives. Only data on hospitalization due to mental disorder were available. However, since mental disorders are often subclinical or treated in out-patient care and suicidality generally increases the probability of being hospitalized for a mental disorder, this could have induced bias.

The familial aggregation of completed suicide was influenced by substantial genetic and also shared 
environmental factors. But what are the possible mechanisms? The shared genetic component could involve liability to impulsive aggression, a propensity to react with aggression or hostility when frustrated or provoked (Turecki, 2001; Brent \& Mann, 2006; McGirr \& Turecki, 2007; Brent \& Melhem, 2008). This should also be reflected in the familial aggregation of violence directed towards others, such as violent crime (Frisell et al. 2011). Personality traits including neuroticism or neurodevelopmental vulnerabilities involving impaired working memory or executive functioning resulting in poor problem solving could also be involved (Baud, 2005; Brent \& Melhem, 2008; Mann et al. 2009). Gene variants related, for example, to the serotonergic-, noradrenergic- and dopaminergic neurotransmitter systems appear to be associated with the risk of suicidal behaviour (Brezo et al. 2008; Currier \& Mann, 2008; Ernst et al. 2009); interaction patterns (gene-gene, gene-development, gene-environment), however, are largely unknown (Rujescu et al. 2007; Brezo et al. 2008, 2010; Currier \& Mann, 2008; Ernst et al. 2009; Roy et al. 2009; Wasserman et al. 2010; Fergusson et al. 2011). The genetic effect probably involves a large number of single genes or alleles, similar to what has been suggested for schizophrenia and bipolar disorder (Purcell et al. 2009). However, it is important to note that such genetic contributions do not rule out that well-designed, environment-based interventions might reduce suicide risk.

On the other hand, our findings also suggested shared environmental effects on familial clustering of completed suicide. This could involve social learning of inadequate handling or communication of frustration or other negative affects, including those following bereavement of a spouse or relative from suicide (Agerbo, 2005; Brent \& Mann, 2006). Transmission of adverse rearing environments could also be involved in the aggregation of suicide among parents and their children (Dinwiddie et al. 2000; Roy, 2002; Brent \& Melhem, 2008). Direct role modelling or imitation of self-destructive behaviours, for instance, between siblings, is also possible although previous studies suggest that this effect is unlikely to be substantial (Statham et al. 1998; Brent \& Melhem, 2008; Burke et al. 2010).

Regarding future research, large epidemiological studies with good statistical power are clearly needed, since suicide is a rare event, even among relatives of suicide victims. Various strategies to gain more specific knowledge on how gene variants, development and environment are associated with suicidal behaviour have been proposed. These include: (a) refining the phenotype for suicidal behaviour; (b) considering different possible pathways to suicidal behaviour. Concerning the former, endophenotypes (intermediate phenotypes between genes and a more overt phenotype or outcome), such as certain personality traits or altered neurocognitive function, should be characterized for suicidal behaviour (Brezo et al. 2008; Mann et al. 2009). Concerning the latter, several possible aetiological pathways for suicidal behaviour have been suggested, for instance, direct effects from psychiatric disorders, effects of early adversity, geneenvironment correlation (selection into adversity), interactions between culture and genes and causal loops from genes to environment and back again (Kendler, 2010), These two overlapping approaches, as well as other strategies, are likely to require further development of technology (e.g. neuroimaging and -physiology) for more fine-grained assessment of biological mechanisms (Wasserman et al. 2010).

To conclude, this study provided strong evidence on the familial aetiology of completed suicide, primarily caused by genetic but also by shared environmental factors. The results confirm the importance of considering the family history of suicide when assessing suicide risk in clinical practice or when designing and administering preventive interventions.

\section{Acknowledgments}

This study was funded by Stockholm County Council, Karolinska Institutet, the Swedish Research Council (grant 2004-4770) and the Swedish Prison and Probation Service R\&D. N.L. was supported by the Swedish Research Council-Medicine (grant 20078595). The funding bodies were not involved in the design and conduct of the study, collection, management, analysis and interpretation of data, or preparation, review or approval of the manuscript.

\section{Declaration of Interest}

None.

\section{References}

Agerbo E (2005). Midlife suicide risk, partner's psychiatric illness, spouse and child bereavement by suicide or other modes of death: a gender specific study. British Medical Journal 59, 407.

Agerbo E, Nordentoft M, Mortensen PB (2002). Familial, psychiatric, and socioeconomic risk factors for suicide in young people: nested case-control study. British Medical Journal 325, 74.

Baldessarini RJ, Hennen J (2004). Genetics of suicide: an overview. Harvard Review of Psychiatry 12, 1-13.

Baud P (2005). Personality traits as intermediary phenotypes in suicidal behavior: genetic issues. American Journal of Medical Genetics. Part C, Seminars in Medical Genetics 133C, $34-42$. 
Bertolote JM, Fleischmann A (2005). Suicidal behavior prevention: WHO perspectives on research. American Journal of Medical Genetics. Part C, Seminars in Medical Genetics 133C, 8-12.

Bondy B, Buettner A, Zill P (2006). Genetics of suicide. Molecular Psychiatry 11, 336-351.

Brent D (2010). What family studies teach us about suicidal behavior: implications for research, treatment, and prevention. European Psychiatry 25, 260-263.

Brent DA, Bridge J, Johnson BA, Connolly J (1996). Suicidal behavior runs in families. A controlled family study of adolescent suicide victims. Archives of General Psychiatry 53, 1145-1152.

Brent DA, Mann JJ (2005). Family genetic studies, suicide, and suicidal behavior. American Journal of Medical Genetics. Part C, Seminars in Medical Genetics 133C, 13-24.

Brent DA, Mann JJ (2006). Familial pathways to suicidal behavior - understanding and preventing suicide among adolescents. New England Journal of Medicine 355, 2719-2721.

Brent DA, Melhem N (2008). Familial transmission of suicidal behavior. Psychiatric Clinics of North America 31, 157-177.

Brezo J, Bureau A, Merette C, Jomphe V, Barker ED, Vitaro F, Hebert M, Carbonneau R, Tremblay RE, Turecki G (2010). Differences and similarities in the serotonergic diathesis for suicide attempts and mood disorders: a 22-year longitudinal gene-environment study. Molecular Psychiatry 15, 831-843.

Brezo J, Klempan T, Turecki G (2008). The genetics of suicide: a critical review of molecular studies. Psychiatric Clinics of North America 31, 179-203.

Burke AK, Galfalvy H, Everett B, Currier D, Zelazny J, Oquendo MA, Melhem NM, Kolko D, HarkavyFriedman JM, Birmaher B, Stanley B, Mann JJ, Brent DA (2010). Effect of exposure to suicidal behavior on suicide attempt in a high-risk sample of offspring of depressed parents. Journal of the American Academy of Child and Adolescent Psychiatry 49, 114-121.

Currier D, Mann JJ (2008). Stress, genes and the biology of suicidal behavior. Psychiatric Clinics of North America 31, 247-269.

Dinwiddie S, Heath AC, Dunne MP, Bucholz KK, Madden PA, Slutske WS, Bierut LJ, Statham DB, Martin NG (2000). Early sexual abuse and lifetime psychopathology: a co-twin-control study. Psychological Medicine 30, 41-52.

Ernst C, Mechawar N, Turecki G (2009). Suicide neurobiology. Progress in Neurobiology 89, 315-333.

Fergusson DM, Horwood LJ, Miller AL, Kennedy MA (2011). Life stress, 5-HTTLPR and mental disorder: findings from a 30-year longitudinal study. British Journal of Psychiatry 198, 129-135.

Frisell T, Lichtenstein P, Långström N (2011). Violent crime runs in families: a total population study of 12.5 million individuals. Psychological Medicine 41, 97-105.

Frisell T, Pawitan Y, Långström N, Lichtenstein P (in press). Heritability, assortative mating and gender differences in violent crime: results from a total population sample using twin, adoption, and sibling models. Behavior Genetics.
Fu Q, Heath AC, Bucholz KK, Nelson EC, Glowinski AL, Goldberg J, Lyons MJ, Tsuang MT, Jacob T, True MR, Eisen SA (2002). A twin study of genetic and environmental influences on suicidality in men. Psychological Medicine 32, 11-24.

Glowinski AL, Bucholz KK, Nelson EC, Fu Q, Madden PA, Reich W, Heath AC (2001). Suicide attempts in an adolescent female twin sample. Journal of the American Academy of Child and Adolescent Psychiatry 40, 1300-1307.

Hawton K, van Heeringen K (2009). Suicide. Lancet 373, 1372-1381.

Kendler KS (2010). Genetic and environmental pathways to suicidal behavior: reflections of a genetic epidemiologist. European Psychiatry 25, 300-303.

Kety SS, Rosenthal D, Wender PH, Schulsinger F (1968). The types and prevalence of mental illness in the biological and adoptive families of adopted schizophrenics. Journal of Psychiatric Research 6, 345-362.

Kim CD, Seguin M, Therrien N, Riopel G, Chawky N, Lesage AD, Turecki G (2005). Familial aggregation of suicidal behavior: a family study of male suicide completers from the general population. American Journal of Psychiatry 162, 1017-1019.

Lichtenstein P, Yip BH, Björk C, Pawitan Y, Cannon TD, Sullivan PF, Hultman CM (2009). Common genetic determinants of schizophrenia and bipolar disorder in Swedish families: a population-based study. Lancet 373, 234-239.

Ludvigsson JF, Otterblad-Olausson P, Pettersson BU, Ekbom A (2009). The Swedish personal identity number: possibilities and pitfalls in healthcare and medical research. European Journal of Epidemiology 24, 659-667.

McGirr A, Alda M, Seguin M, Cabot S, Lesage A, Turecki G (2009). Familial aggregation of suicide explained by cluster B traits: a three-group family study of suicide controlling for major depressive disorder. American Journal of Psychiatry 166, 1124-1134.

McGirr A, Turecki G (2007). The relationship of impulsive aggressiveness to suicidality and other depression-linked behaviors. Current Psychiatry Reports 9, 460-466.

Magnusson PK, Gunnell D, Tynelius P, Davey Smith G, Rasmussen F (2005). Strong inverse association between height and suicide in a large cohort of Swedish men: evidence of early life origins of suicidal behavior? American Journal of Psychiatry 162, 1373-1375.

Mann JJ, Arango VA, Avenevoli S, Brent DA, Champagne FA, Clayton P, Currier D, Dougherty DM, Haghighi F, Hodge SE, Kleinman J, Lehner T, McMahon F, Moscicki EK, Oquendo MA, Pandey GN, Pearson J, Stanley B, Terwilliger J, Wenzel A (2009) Candidate endophenotypes for genetic studies of suicidal behavior. Biological Psychiatry 65, 556-563.

Mann JJ, Bortinger J, Oquendo MA, Currier D, Li S, Brent DA (2005). Family history of suicidal behavior and mood disorders in probands with mood disorders. American Journal of Psychiatry 162, 1672-9.

Mittendorfer-Rutz E, Rasmussen F, Wasserman D (2004). Restricted fetal growth and adverse maternal psychosocial and socioeconomic conditions as risk factors for suicidal 
behaviour of offspring: a cohort study. Lancet 364, 1135-1140.

Mittendorfer-Rutz E, Rasmussen F, Wasserman D (2008). Familial clustering of suicidal behaviour and psychopathology in young suicide attempters. A register-based nested case control study. Social Psychiatry and Psychiatric Epidemiology 43, 28-36.

National Board of Health and Welfare (2010). Causes of death 2008. National Board of Health and Welfare: Stockholm, Sweden.

Neeleman J, Wessely S (1997). Changes in classification of suicide in England and Wales: time trends and associations with coroners' professional backgrounds. Psychological Medicine 27, 467-472.

Pedersen NL, Fiske A (2010). Genetic influences on suicide and nonfatal suicidal behavior: twin study findings. European Psychiatry 25, 264-267.

Purcell SM, Wray NR, Stone JL, Visscher PM, O'Donovan MC, Sullivan PF, Sklar P (2009). Common polygenic variation contributes to risk of schizophrenia and bipolar disorder. Nature 460, 748-752.

Qin P, Agerbo E, Mortensen PB (2002). Suicide risk in relation to family history of completed suicide and psychiatric disorders: a nested case-control study based on longitudinal registers. Lancet 360, 1126-1130.

Qin P, Agerbo E, Mortensen PB (2003). Suicide risk in relation to socioeconomic, demographic, psychiatric, and familial factors: a national register-based study of all suicides in Denmark, 1981-1997. American Journal of Psychiatry 160, 765-772.

Riordan DV, Selvaraj S, Stark C, Gilbert JS (2006). Perinatal circumstances and risk of offspring suicide. Birth cohort study. British Journal of Psychiatry 189, 502-507.

Roy A (2002). Childhood trauma and neuroticism as an adult: possible implication for the development of the common psychiatric disorders and suicidal behaviour. Psychological Medicine 32, 1471-1474.

Roy A, Rylander G, Sarchiapone M (1997). Genetics of suicides. Family studies and molecular genetics. Annals of the New York Academy of Sciences 836, 135-157.

Roy A, Sarchiopone M, Carli V (2009). Gene-environment interaction and suicidal behavior. Journal of Psychiatric Practice 15, 282-288.

Rujescu D, Thalmeier A, Moller HJ, Bronisch T, Giegling I (2007). Molecular genetic findings in suicidal behavior: what is beyond the serotonergic system? Archives of Suicide Research 11, 17-40.

Runeson B, Åsberg M (2003). Family history of suicide among suicide victims. American Journal of Psychiatry 160, $1525-1526$.

Runeson BS (1998). History of suicidal behaviour in the families of young suicides. Acta Psychiatrica Scandinavica 98, 497-501.

Schulsinger F, Kety SS, Rosenthal D, Wender PH (1979). A family study of suicide. In Origins, Prevention, and Treatment of Affective Disorders (ed. S. E. Schou), pp. 277-87. Academic: New York.

Segal NL (2009). Suicidal behaviors in surviving monozygotic and dizygotic co-twins: is the nature of the co-twin's cause of death a factor? Suicide and Life-Threatening Behavior 39, 569-575.

Statham DJ, Heath AC, Madden PA, Bucholz KK, Bierut L, Dinwiddie SH, Slutske WS, Dunne MP, Martin NG (1998). Suicidal behaviour: an epidemiological and genetic study. Psychological Medicine 28, 839-855.

Turecki G (2001). Suicidal behavior: is there a genetic predisposition? Bipolar Disorders 3, 335-349.

Wasserman D, Geijer T, Sokolowski M, Rozanov V, Wasserman J (2007). Nature and nurture in suicidal behavior, the role of genetics: some novel findings concerning personality traits and neural conduction. Physiology \& Behavior 92, 245-249.

Wasserman D, Terenius L, Wasserman J, Sokolowski M (2010). The 2009 Nobel conference on the role of genetics in promoting suicide prevention and the mental health of the population. Molecular Psychiatry 15, $12-17$.

Wender PH, Kety SS, Rosenthal D, Schulsinger F, Ortmann J, Lunde I (1986). Psychiatric disorders in the biological and adoptive families of adopted individuals with affective disorders. Archives of General Psychiatry 43, 923-929.

von Borczyskowski A, Lindblad F, Vinnerljung B, Reintjes R, Hjern A (2010). Familial factors and suicide: an adoption study in a Swedish National Cohort. Psychological Medicine, 1-10.

Voracek M, Loibl LM (2007). Genetics of suicide: a systematic review of twin studies. Wiener Klinische Wochenschrift 119, 463-475.

Voracek M, Loibl LM (2008). Consistency of immigrant and country-of-birth suicide rates: a meta-analysis. Acta Psychiatrica Scandinavica 118, 259-271. 
Appendix. Relative risks of suicidal behaviour (completed suicide or suicide attempt leading to hospital care) in relatives of all probands who committed suicide in Sweden during 1952-2003 ( $n=83$ 951) compared with relatives of matched controls, in a total population cohort of 11384649 individuals

\begin{tabular}{|c|c|c|c|}
\hline Relation to proband & $\begin{array}{l}\text { Number } \\
\text { of dyads }\end{array}$ & $\begin{array}{l}\text { Concordant } \\
\text { pairs }\end{array}$ & $\begin{array}{l}\text { Matched odds } \\
\text { ratio }(95 \% \mathrm{CI})\end{array}$ \\
\hline \multicolumn{4}{|l|}{ First-degree relatives } \\
\hline Child & 13714253 & 4689 & $2.1(2.0-2.1)$ \\
\hline Adopted-away child & 66564 & 87 & $1.6(1.2-2.0)$ \\
\hline \multicolumn{4}{|l|}{ Second-degree relatives } \\
\hline Maternal half-sibling & 1121888 & 246 & $1.5(1.3-1.7)$ \\
\hline Paternal half-sibling & 1482456 & 219 & $1.1(0.9-1.2)$ \\
\hline \multicolumn{4}{|l|}{ Non-biological relatives } \\
\hline Adopted child & 206692 & 75 & $1.6(1.2-2.0)$ \\
\hline \multicolumn{4}{|l|}{ Twins } \\
\hline Twin (monozygotic) & 14018 & 12 & $12.3(6.5-23.2)$ \\
\hline Twin (dizygotic, same-gender) & 19664 & 7 & $2.3(1.1-5.0)$ \\
\hline
\end{tabular}

CI, Confidence interval.

Relative risks in this table were calculated by the same procedure as those in Table 1. 